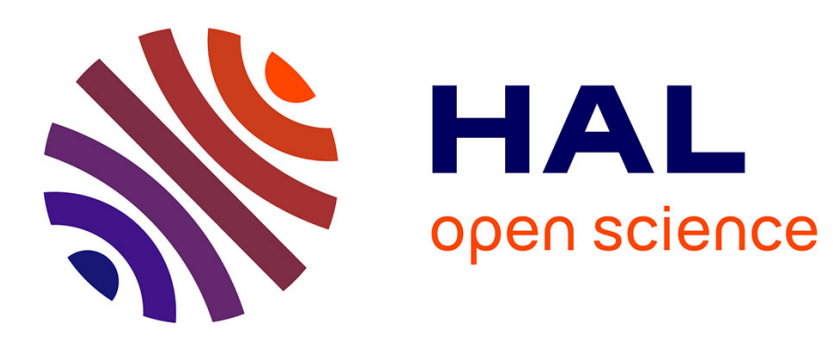

\title{
Multimodal beam vibration damping exploiting PZT transducers and passive distributed circuits
}

\author{
Maurizio Porfiri, Francesco Dell'Isola
}

\section{To cite this version:}

Maurizio Porfiri, Francesco Dell'Isola. Multimodal beam vibration damping exploiting PZT transducers and passive distributed circuits. Journal de Physique IV Proceedings, 2004, pp.8. hal-00497103

\section{HAL Id: hal-00497103 \\ https://hal.science/hal-00497103}

Submitted on 2 Jul 2010

HAL is a multi-disciplinary open access archive for the deposit and dissemination of scientific research documents, whether they are published or not. The documents may come from teaching and research institutions in France or abroad, or from public or private research centers.
L'archive ouverte pluridisciplinaire HAL, est destinée au dépôt et à la diffusion de documents scientifiques de niveau recherche, publiés ou non, émanant des établissements d'enseignement et de recherche français ou étrangers, des laboratoires publics ou privés. 


\title{
Multimodal beam vibration damping exploiting PZT transducers and passive distributed circuits
}

\author{
M. Porfiri ${ }^{1}$ and F. dell'Isola ${ }^{2}$ \\ 1 Dipartimento di Meccanica ed Aeronautica, Universita di Roma "La Sapienza", \\ Via Eudossiana 18, 184 Roma, Italy \\ 2 Dip. Ing. Strutt. \& Geotecnica, Univ. “La Sapienza” Via Eudossiana 18, 184 Rome, Italy
}

\begin{abstract}
Structures are subject to extensive vibrations that can reduce their life and contribute to failure. Piezoelectric transducers in conjunction with appropriate electric networks, can be used as a mechanical energy dissipation device. Most of the previous work has focused on active control in which electric power is supplied to the actuators that exert actions on the host structure to suppress its vibrations. Alternatively, undesired mechanical energy of a host structure could be converted into electrical energy that can be dissipated through a set of resistor. This does not require an external power unit and is a more economical means of controlling vibrations of a structure, but an effective transduction of mechanical energy into electric energy has to be guaranteed. Therefore, in the present work a completely passive distributed electric controller is found to achieve simultaneous beam multimodal vibration suppression. The optimal electric network (interconnecting the piezoelectric transducers) may be synthesized as a finite differences approximation of the derived distributed (infinite dimensional) optimal controller.
\end{abstract}

\section{INTRODUCTION}

In [1] the possibility of damping beam vibrations by means of a piezoelectric transducer positioned on the structure and shunted with an $(L R)$ impedance is investigated. The passive impedance combined with the inherent capacitance of the piezoelectric transducer creates a damped electromechanical beating. The method proposed in [1] allows for an efficient single-mode control of structural vibrations, whenever the resonant circuit is tuned to the mechanical mode to be suppressed; nevertheless the efficiency of the electromechanical coupling strongly depends on the position of the transducer over the host structure. Moreover, the technical feasibility of the passive piezoelectric controller proposed in [1] is limited, since too large inductors are required to produce low-frequency electrical resonance with the small inherent capacitance of the piezoelectric transducer. A generalization of the "piezoelectric shunt damping" technique proposed in [1] to the multimodal control of vibrations is presented in [2] and [3], where complicated impedances are placed across the terminals of the piezoelectric transducer. Such a control methodology seems to present severe inconveniences, indeed the used inductances are still very high and the damping efficiency, even for few modes, is drastically reduced.

In order to cure these drawbacks, without forsaking the advantages featured by passive control, in [4] it is proposed to position an array of piezoelectric elements on the beam and interconnect their electric terminals via a circuit "analog" to the beam (i.e. governed by the same evolution equations) completed with proper resistors. The electric analogs synthesized in [4] show several inconveniences, 
e.g. the presences of negative inductors and multiport transformers. In order to overcome these technical problems, in [5] an electric analog exploiting only inductors and single port transformers is derived.

In both [4] and [5], it is a priori assumed that, in order to achieve effective damping of several modes, the electric interconnection should be the beam electric analog. The aim of this paper is to substantiate this assumption by proving that the electric analog represents the optimal passive controller. Indeed, we will consider a rather wide class of passive electric controllers and we will formulate and solve the problem of finding the optimal controller evolution equations in order to maximize the time decay rates of every mechanical mode.

Section 2 is devoted to the derivation of the equations of motion of a Piezo-ElectroMechanical ( $\mathbb{P E M}$ for brevity) beam, i.e. a beam coupled to a passive electric network via distributed piezoelectric transduction. The beam is modelled as a continuous system, while the electric circuit is regarded as a lumped system. From the mechanical point of view, the piezoelectric elements introduce additional stiffness and mass, and exert electrically driven couples at their ends; while, from the electric point of view they are treated as capacitors in parallel connection with current sources driven by average beam strain rates. Therefore, the resulting system state is ruled by the beam deflection field and the discrete set of circuit independent flux linkages. In previous works, e.g. [6], no investigations were made towards unstructured passive electric networks, i.e. circuits with not prescribed topology (in [6] several electric interconnections constituted solely by two terminal impedances are analyzed). The resulting governing equations cannot be easily handled without exploiting numerical techniques.

In order to achieve a more maniable model of the overall system, in Section 3 a homogenized model of a $\mathbb{P E M}$ beam is deduced from the previously introduced refined model. The state of the homogenized system is determined by the beam deflection field and the electric flux linkage field, and its motion is governed by two gyroscopically coupled partial differential equations. In order to avoid spillover phenomena in between different mechanical modes, the electric operators appearing in the governing equations are assumed to share the mechanical eigenfunctions; namely, their eigenfunctions are assumed to be equal to the mechanical ones and only their eigenvalues are left as unknowns.

Considering the simply supported case, the modal analysis of the $\mathbb{P E M}$ beam can be easily performed as it is done in Section 4. In particular, a simple expression for the eigenvalues of the $\mathbb{P E M}$ beam is derived.

In Section 5 the optimization problem is formulated and completely solved, by the use of the aforementioned modal analysis. Indeed, the hypothesis made on the eigenfunctions of the electric operators, i.e. to be coincident with the mechanical modal shapes, together with the modal expansion worked in Section 4 lead to the simpler problem of optimizing a sequence of decoupled finite dimensional controllers. The solution of these standard problems enables the determination and spectral representation of the optimal electric operators. In particular it is proven that the optimal interconnection is the "analog" one, completed with resistors represented by a second order spatial derivative.

\section{REFINED MODEL FOR PEM BEAMS}

We consider a host beam of length $l$, width $w$ and thickness $h$ on which an array of Nuniformly distributed piezoelectric transducers is positioned as shown in Figure 1 (covering both the beam faces). The length of the transducers is assumed to be equal to $l_{p}$, while the width is assumed to be equal to that of the beam; $d$ denotes the distance between the adjacent patches.

The piezotransducers are placed along the beam axis and polarized in the transverse direction, in the so called bender configuration (see e.g. [5]). These transducers will be interconnected by an electrical network which will be synthesized in order to accomplish given optimality conditions on the mechanical vibration decay. 


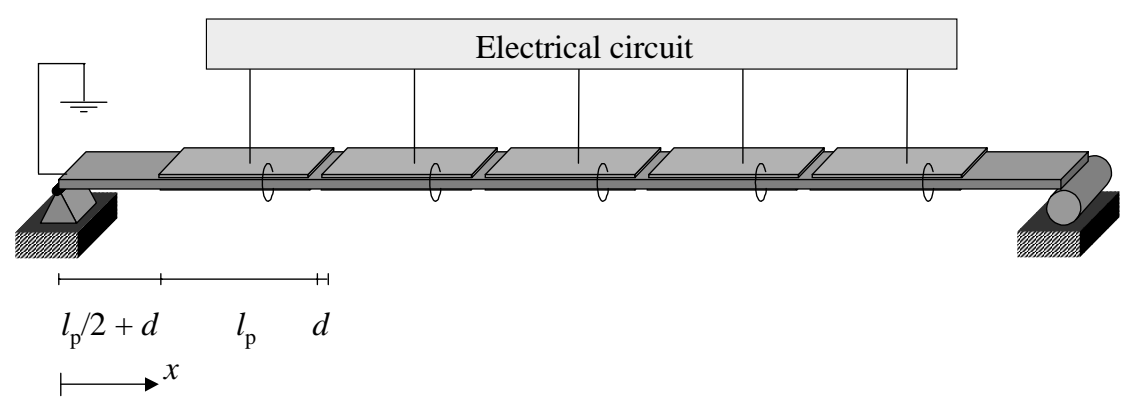

Figure 1. Geometry of the problem.

Introducing a set of nodes $\{x i\}_{i=1, \ldots, N}$ representing the geometrical centers of the transducers, defined by:

$$
x_{i}=\left(l_{p}+d\right) i \quad i=1, \ldots N
$$

and also including the boundary nodes $x_{0}=0$ and $x_{N+1}=l$, the contact couple $M$ at the section labelled by the abscissa $x$, over the beam span, can be expressed as the sum of mechanical and piezoelectric contributions as follows:

$$
M(x, t)=\left[E_{b} I_{b}+k_{m m} \sum_{i=1}^{N}\left(R E C T_{l p}\left(x-x_{i}\right)\right)\right] u^{\prime \prime}(x, t)+k_{m e} \sum_{i=1}^{N}\left(R E C T_{l p}\left(x-x_{i}\right) \dot{\psi}(l)\right),
$$

where $\operatorname{RECT}_{l p}\left(x-x_{i}\right)$ is a unit step function centered at $x_{i}$ of width $l_{p}, \psi_{i}$ is the flux linkage of the $i$-th transducer measured with respect to a common reference ground for every transducer, $u$ is the beam deflection field, $\mathrm{E}_{b}$ is the Young modulus of the material of the beam, $\mathrm{I}_{b}$ is the beam section moment of inertia, $k_{m m}$ is the transducers bending stiffness, $k_{m e}$ is the piezoelectric coupling coefficient, $t$ denotes the time variable and superposed dot and prime respectively mean time and space derivative. The distributed inertia can be accounted for by introducing the following constitutive equation for the applied load (external mechanical forcing is excluded):

$$
b_{T}(x, t)=-\left(\rho_{b}+\sum_{i=1}^{N}\left(R E C T_{l p}\left(x-x_{i}\right)\right) \rho_{p}\right) \ddot{u}(x, t) .
$$

where $\rho_{b}$ and $\rho_{p}$ are respectively the mass density per unit length of the beam and the transducer layers.

The balance equations for the considered electrically excited vibrating beam yield:

$$
M(x, t)^{\prime \prime}-b_{T}(x, t)=0
$$

when $M(x, t)$ and $b_{T}(x, t)$ are given by Equations (2) and (3) respectively.

From a purely electrical point of view the $i$-th piezoelectric bender transducer can be described as a capacitor kee in parallel connection with a "mechanically driven" current source, which injects into the electrical circuit the current $J_{i}$ driven by its mechanical time rate of deformation:

$$
J_{i}=k_{m e}\left(\dot{u}^{\prime}\left(x_{i}+l_{p} / 2, t\right)-\dot{u}^{\prime}\left(x_{i}-l_{p} / 2, t\right)\right) .
$$

The electrical system interconnecting the electrical terminals of the bender transducers is assumed to be a linear, time invariant, reciprocal, passive $N$ terminals network (see e.g. [7]) constituted only of 
resistors, inductors and transformers. Thus by Kirchhoff balance of currents at node $i$, the evolution equations for the variables $\psi_{j}$ are given by the following second order system of ODEs:

$$
k_{e e} \ddot{\psi}_{i}+\sum_{j=1}^{N} K_{i j} \psi_{j}+\sum_{j=1}^{N} D_{i j} \dot{\psi}_{j}-k_{m e}\left(\dot{u}^{\prime}\left(x_{i}+l_{p} / 2, t\right)-\dot{u}^{\prime}\left(x_{i}-l_{p} / 2, t\right)\right)=0, \quad i=1, \ldots N .
$$

$\left[K_{i j}\right]$ being the electric stiffness and $\left[D_{i j}\right]$ the electric dissipation.

\section{A CLASS OF HOMOGENIZED MODELS FOR PEM BEAMS}

When it can be assumed that the number of piezoelectric transducers is sufficiently large, a continuous flux linkage field $\psi(x, t)$ can be introduced and the governing equation expressed in terms of the deflection $u$ is seen to be (for more details on the needed homogenization techniques see e.g. [8]):

$$
k_{M} u^{I V}+\rho \ddot{u}+c_{f} K_{m e} \dot{\psi}^{\prime \prime}=0, \quad\left\{\begin{array}{c}
k_{M}=E_{b} I_{b}+c f k_{m m} \\
\rho=\rho b+c f \rho_{p}
\end{array}\right.
$$

where the covering factor is defined as:

$$
c_{f}=\frac{1}{1+d / l_{p}} .
$$

Thus the dimensionless form of the mechanical evolution equation becomes:

$$
\ddot{u}+\alpha^{4} u^{I V}+\beta_{e m}^{2} \dot{\psi}^{\prime \prime}=0, \quad\left\{\begin{array}{c}
\alpha^{4}=\frac{K_{M}}{l^{A} \rho \omega_{0}^{2}} \\
\beta_{e m}^{2}=\frac{c_{f} k_{m e} \psi_{0}}{l^{2} \rho \omega_{0} u_{0}}
\end{array},\right.
$$

where a characteristic frequency $\omega_{0}$, a characteristic length equal to the beam length $l$, a characteristic deflection $u_{0}$ and a characteristic flux linkage $\psi_{0}$ have been introduced. As this cannot cause misunderstanding, we have adopted the same letters to denote both dimensional and dimensionless differential operators and kinematical descriptors.

Furthermore, when the number of transducers can be assumed to be sufficiently large, the electrical system can be described by a sole PDE which, expressed in terms of the dimensionless flux-linkage $\psi$, reads:

$$
\ddot{\psi}+\mathbf{K}[\psi]+\mathbf{D}[\dot{\psi}]-\beta_{m e}^{2} \dot{u}^{\prime \prime}=0, \quad \beta_{m e}^{2}=\frac{k_{m e} u_{0}}{l^{2}\left(\frac{k_{e e}}{l_{p}}\right) \omega_{0} \psi_{0}},
$$

where $\mathbf{K}$ and $\mathbf{D}$ are linear operators. In the following we will restrict our investigation to simply supported beams $\mathbb{P E M}$ beams, i.e. we will assume simply supporting boundary conditions for both the mechanical and electrical systems. Furthermore, in order to avoid undesired spillover phenomena in between different vibration modes, we will look for a dissipative electric network which does not modify the electrical and mechanical modal shapes. Therefore, in the present paper the following restrictive assumption on $\mathbf{K}$ and $\mathbf{D}$ are made:

$$
\mathbf{K}[\cdot]=k\left((\cdot)^{I V}\right), \quad \mathbf{D}[\cdot]=d\left((1)^{I V}\right)
$$

$k$ and $d$ being analytic functions which take positive values on the real positive axis ${ }^{\mathrm{a}}$, see e.g. [9].

${ }^{\text {a }}$ The positiveness of $k(\lambda)$ and $d(\lambda)$, when $\lambda$ is positive assures the passivity of the electric network. 
This hypothesis on the structure of the electrical stiffness and damping, respectively $\mathbf{K}$ and $\mathbf{D}$, seems to be general enough for our design aims and leads to a simple solution of the synthesis problem of determining an optimal lumped electrical circuit to be piezoelectrically coupled to the given flexible structure (see e.g. [4] and [5]). Thus the dimensionless form of the evolution electromechanical equations is:

$$
\left\{\begin{array}{c}
\ddot{u}+\alpha^{4} u^{I V}+\beta^{2} \dot{\psi}^{\prime \prime}=0 \\
\ddot{\psi}+\mathbf{K}[\psi]+\mathbf{D}[\dot{\psi}]-\beta^{2} \dot{u}^{\prime \prime}=0
\end{array} \quad ; \quad\left\{\begin{array}{c}
\alpha^{4}=\frac{K_{M}}{l^{4} \omega_{0}^{2}} \\
\beta^{2}=\frac{k_{m e}}{l^{2} \omega_{0}} \sqrt{\frac{c_{f} l_{p}}{k_{e e} \rho}}
\end{array}\right.\right.
$$

where, in order to preserve the form of a gyroscopic coupling,the characteristic flux linkage and displacement are chosen to satisfy the following relation:

$$
\sqrt{\frac{c_{f} K_{e e}}{l_{p} \rho}}=\frac{u_{0}}{\psi_{0}} .
$$

The eigenfunctions of the both the mechanical and electrical operators are easily found to be:

$$
v_{m}(x)=\sqrt{2} \sin (m \pi x), \quad m=1,2, \ldots ;
$$

The eigenvalues of the elastica operator are

$$
(m \pi)^{4}, \quad m=1,2, \ldots ;
$$

while those of the electric stiffness and dissipation operators are respectively $k(m)$ and $d(m)$.

\section{MODAL ANALYSIS OF HOMOGENIZED $\mathbb{P E M}$ BEAMS}

In this subsection a modal analysis for a simply-supported $\mathbb{P E M}$ beam, based upon the Galerkin method, is performed.

The set of basis functions chosen to represent the deflection $u(x, t)$ and the flux linkage $\rho(x, t)$ of the $\mathbb{P E M}$ beam is constituted by the eigenfunctions $v_{m}(x)$ specified in (9) of the elastica differential operator $(\cdot)^{I V}$ determining the modal forms for both the uncoupled electrical and mechanical motions (9). Consequently it is useful to consider a Fourier expansion for the solution of (8):

$$
\left[\begin{array}{l}
u(x, t) \\
\psi(x, t)
\end{array}\right]=\sum_{m=1}^{\infty}\left[\begin{array}{l}
p_{m}(t) \\
q_{m}(t)
\end{array}\right] \quad v_{m}(x),
$$

in terms of the mechanical and electrical Fourier coefficients $p_{m}(t)$ and $q_{m}(t)$ of the expansion; achieving with simple algebraic manipulations the following system of $\mathrm{ODE}_{s}$ governing their time evolution, in which unitary dimensionless modal masses are made to appear:

$$
\begin{aligned}
& {\left[\begin{array}{ll}
1 & 0 \\
0 & 1
\end{array}\right]\left[\begin{array}{l}
\ddot{p}_{m}(t) \\
\ddot{q}_{m}(t)
\end{array}\right]+\left[\begin{array}{cc}
\omega_{p}^{2}(m) & 0 \\
0 & \omega_{q}^{2}(m)
\end{array}\right]\left[\begin{array}{l}
p_{m}(t) \\
q_{m}(t)
\end{array}\right]+\left[\begin{array}{cc}
0 & -\sqrt{c(m)} \\
\sqrt{c(m)} & 2 \zeta_{q}(m) \omega_{q}(m)
\end{array}\right] \quad\left[\begin{array}{l}
\dot{p}_{m}(t) \\
\dot{q}_{m}(t)
\end{array}\right]=\left[\begin{array}{l}
0 \\
0
\end{array}\right] } \\
&\left\{\begin{array}{l}
\omega_{p}^{2}(m)=(\alpha m \pi)^{4} \\
\omega_{q}^{2}(m)=k(m) \\
\zeta_{q}(m)=\frac{1}{2} \frac{d(m)}{2 \sqrt{k(m)}} \\
c(m)=(\beta m \pi)^{4}
\end{array}\right.
\end{aligned}
$$


Let us remark that $\omega_{p}(m)$ and $\omega_{q}(m)$ respectively the natural angular frequency of the mechanical and electrical $m$-th modes, while $c(m)$ denotes the $m$-th gyroscopic coupling coefficient and $\zeta_{q}(m)$ the damping factor of the $m$-th electrical mode. Let us explicitly remark that the chosen boundary conditions and basis functions lead to a set of uncoupled evolution problems for each electromechanical pair of Fourier coefficients, hence it is possible to easily derive an analytical solution for each $m$-th pair. The poles of the system in (10) can be determined as the four zeros $s_{1}^{m}, \ldots s_{4}^{m}$ of the following polynomial:

$$
\begin{aligned}
P^{m}(s)=s^{4}+2 \zeta_{q}(m) w_{q}(m) s^{3}+\left(w_{q}^{2}(m)+c(m)+\right. & \left.w_{q}^{2}(m)\right) s^{2}+ \\
& +2 \zeta_{q}(m) \omega_{q}(m) \omega_{p}^{2}(m) s+\omega_{p}^{2}(m) \omega_{q}^{2}(m) .
\end{aligned}
$$

\section{DESIGN OF THE PASSIVE OPTIMAL CONTROLLER}

In this section we will design the optimal passive distributed controller following the pole placement technique applied to the evolution of each pair of electrical and mechanical modes. In particular, we will determine an optimal expression for the stiffness and dissipative functions $k(m)$ and $d(m)$ appearing in the time evolution of the $m$-th electrical mode; consequently, we will establish the forms of the operators $\mathbf{K}$ and $\mathbf{D}$, exploiting their spectral representation.

The chosen optimality condition requires the determination of the values of $\omega_{q}(m)$ and $\zeta_{q}(m)$ in order to maximize the exponential time decay rate $\tau^{m}$ of the solution of the fourth order system (10), governing the time evolution of the $m$-th pair of electrical and mechanical modes, defined as:

$$
\tau^{m}=\min _{i=1, \ldots 4}\left\{\left|\operatorname{Re}\left[s_{i}^{m}\right]\right|\right\} .
$$

In order to maximize the exponential time decay $\tau^{m}$, the optimal values of $\omega_{q}(m)$ and $\zeta_{q}(m)$ by requiring the four roots of $P^{m}(s)$ to be coincident (pole placement technique, see e.g. [1]). Hence we enforce the polynomial $P^{m}(s)$ to be factorized as:

$$
P^{m}(s) \equiv\left(s^{2}+2 \sigma(m) s+\left(\sigma(m)^{2}+\omega(m)^{2}\right)\right)^{2} .
$$

Equating the coefficients of the above mentioned polynomials the following the following matching conditions are imposed on the electrical controller:

$$
\left\{\begin{array}{l}
\omega_{q}(m)=\omega_{p}(m) \\
\zeta_{q}(m)=\frac{\sqrt{c(m)}}{\omega_{p}(m)}
\end{array} .\right.
$$

Condition $(12)_{1}$ establishes that in order to maximize the time rate decay of the $m$-th pair of electrical and mechanical modes, the electrical $m$-th mode has to be resonant at the mechanical resonance frequencyq $\omega_{q}(m)$.

Thus, by use of (11), the following conditions on the values of the functions $k$ and $d$ in correspondence with the mode number $m$ under control are stated:

$$
\left\{\begin{array}{l}
k(m)=(\alpha m \pi)^{4} \\
d(m)=2(\beta m \pi)^{2} .
\end{array}\right.
$$

The values of the real and imaginary part of the coincident roots are found to be:

$$
\left\{\begin{array}{l}
\sigma(m)=\frac{\sqrt{c(m)}}{2}, \\
\omega(m)=\sqrt{\omega_{p}(m)^{2}-\frac{c(m)}{4}}
\end{array}\right.
$$


hence, the damping ratio (defined as the sine of the phase of the coincident roots measured from the imaginary axis) becomes:

$$
\zeta(m):=\frac{\sigma(m)}{\sqrt{\omega(m)^{2}+\sigma(m)^{2}}}=\frac{\sqrt{c(m)}}{2 \omega_{p}(m)} .
$$

Introducing the relations (11) into (14), we get the following expressions for the real and imaginary parts of the placed roots:

$$
\left\{\begin{array}{l}
\sigma(m)=\frac{(\beta m \pi)^{2}}{2} \\
\omega(m)=(m \pi)^{2} \sqrt{\alpha^{4}-\frac{\beta^{4}}{4}}
\end{array}\right.
$$

Taking into account the definitions (8) of the parameters $\alpha$ and $\beta$, we get the following relation in terms of the properties of the piezo-electromechanical beam

$$
\zeta(m)=\frac{c_{f} k_{m e}}{2 \sqrt{\gamma K_{M}}}
$$

Conditions (13) establish that the optimal distributed passive circuit to be mechanically fed by the piezotransducers has to be governed by the following PDE:

$$
\ddot{\psi}+\mathbf{K}[\psi]+\mathbf{D}[\dot{\psi}]=0
$$

where the stiffness and damping operators are defined by:

$$
\left\{\begin{array}{l}
\mathbf{K}[\psi]=\alpha^{4} \psi^{I V} \\
\mathbf{D}[\dot{\psi}]=-2 \beta^{2} \dot{\psi}^{\prime \prime}
\end{array}\right.
$$

Remark that the self-adjoint operators $\mathbf{K}$ and $\mathbf{D}$ are both differential operators and satisfy the relationship

$$
\mathbf{K} \propto \mathbf{D}^{2}
$$

The found electrical circuit can be thought as the analog circuit of a simply supported vibrating beam, with an internal dissipation proportional to the curvature velocity $\dot{u}^{\prime \prime}$. The synthesis of a lumped electrical network governed by a finite difference approximation of (16) with (17) has been addressed in [5] and [4].

\section{CONCLUSIONS}

In the present paper the concept of piezoelectric shunting by means of a single transducer suitably positioned on a structural member is generalized. Indeed we consider a beam hosting an array of piezoelectric transducers and look for a multiterminal electric network shunting them in an optimal way. In the first two sections a maniable model for a $\mathbb{P E M}$ beam is found, while in the subsequent sections its electric components are optimized to get the most efficient damping of mechanical vibrations. We have proved that, in principles, a multishape, multifrequency (i.e. a complete multimodal) vibration suppression is possible by means of a completely passive electric controller. This is obtained by synthesizing a distributed electric circuit governed by the same PDE governing the beam flexural vibrations, and completed with a resistive network governed by the second order spatial derivative. Even if we have considered only a simply supported $\mathbb{P E M}$ beam, the same reasonings can be successfully applied to differently constrained $\mathbb{P E M}$ beams. Nevertheless, in these cases the optimal electric operators may not be purely differential as in (17). 


\section{Acknowledgements}

The partial support of the Engineering Science and Mechanics Department of Virginia Polytechnic Institute and State University is gratefully acknowledged by the authors. This presented research has been also partially supported by MIUR, Ministero per l'Innovazione, l'Università e la Ricerca Fondi Ricerca PRIN "Sintesi di circuiti piezoelettrici e tecniche di disaccoppiamento per il controllo di vibrazioni meccaniche" (protocollo 2001097882_003) and by the Università di Roma "La Sapienza".

\section{References}

[1] Hagood N. W., von Flotow A., "Damping of structural vibrations with piezoelectric materials and passive electrical networks", Journal of Sound and Vibrations 146 (1991) 243-268.

[2] Hollkamp J. J., "Multimodal Passive Vibration Suppression with Piezoelectric Materials and Resonant Shunts", Journal of Intelligent Material Systems and Structures 5 (1994) 49-57.

[3] Fleming A. J., Behrens S., and Moheimani S. O. R., "Optimization and Implementation of Multimode Piezoelectric Shunt Damping Systems", IEEE/ASME Transactions on Mechatronics 7(1) (2002) 87-94.

[4] Alessandroni S., dell'Isola F., Porfiri M., "A revival of electric analogs for vibrating mechanical systems aimed to their efficient control by PZT actuators", International Journal of Solids and Structures, 39(20) (2002) 5295-5324.

[5] Andreaus U., dell'Isola F., and Porfiri M., "Piezoelectric passive distributed controllers for beam flexural vibrations", to appear in Journal of Vibration and Control.

[6] Maurini C, dell'Isola F, Del Vescovo D., "Comparison of piezoelectronics networks acting as distributed vibration absorbers", Mechanical Systems and Signal Processing, in press.

[7] Newcomb R. W., Linear Multiport Synthesis (McGraw Hill, 1966).

[8] Kader M., Lenczner M., and Mrcarica Z., "Distributed control based on distributed electronic circuits: application to vibration control", Microelectronics Reliability 41 (2001) 1857-1866.

[9] Pedersen M., Functional Analysis in Applied Mathematics and Engineering (Chapman \& Hal, 1999). 